\title{
Revisiting the region of the open cluster NGC 5606 $\star \star \star \star$
}

\author{
A. M. Orsatti ${ }^{1,2, \dagger}$, C. Feinstein ${ }^{1,2, \dagger}$, E. I. Vega ${ }^{1,3 \dagger}$, and M. M. Vergne ${ }^{1,2, \dagger}$ \\ ${ }^{1}$ Facultad de Ciencias Astronómicas y Geofísicas (Universidad Nacional de La Plata), Paseo del Bosque s/n, 1900 La Plata, Argentina \\ e-mail: aorsatti@fcaglp.unlp.edu.ar \\ 2 Instituto de Astrofísica de La Plata, (IALP, CONICET), Paseo del Bosque s/n, 1900 La Plata, Argentina \\ 3 Instituto de Astronomía y Física del Espacio, C. C. 67 Suc. 28, 1428 Buenos Aires, Argentina
}

Received 28 December 2006 / Accepted 19 April 2007

\begin{abstract}
We present polarimetric observations in the UBVRI bands corresponding to 54 stars located in the direction of NGC 5606 . Our intention is to analyze the dust characteristics between the Sun and the cluster, as well as to confirm doubtful memberships using polarimetric tools. We also want to determine if a group of 11 B and A stars identified in the past by Vázquez \& Feinstein (1991, A\&AS, 87, 383) in front of NGC 5606 has any physical entity. From polarimetric data we have found at least two dust layers along the line of sight to the open cluster. The observations show that both dust layers have their local magnetic field with an orientation which is close to the direction of the Galactic Plane $\left(\theta=70^{\circ} 0\right)$, but the direction of the polarimetric vector for the members of the cluster seems to be lower than this value. NGC 5606 can be polarimetrically characterized with $P_{\max }=2.97 \%$ and $\theta_{v}=63^{\circ} .5$. The internal dispersion of the polarization values for the members of NGC 5606 seems to be compatible with intracluster dust. We were able to add four possible new members to the list of stars in NGC 5606, and to reject others accepted as members in past investigations; and we identified 17 (out of the 54 observed stars) with intrinsic polarization in their light.

The group of late B- and A-type stars could be physically related, with characteristics of an open cluster of intermediate age (between 0.79 and $1 . \times 10^{8} \mathrm{yr}$ ). For this group we obtained representative values of $P=2.12 \%$ and $\theta_{v}=70^{\circ}$. . The new cluster, which we have provisionally named Anon (La Plata 1), extends from NE to SW across the face of NGC 5606 covering about $10^{\prime}$. The fitting procedure of Schmidt-Kaler's ZAMS (1982, in Landolt/Bornstein, Neue Series VI/2b) was used to derive the distance to the group, obtaining a distance modulus of $V_{o}-M_{v}=9.90$ (649 pc from the Sun). The mean color excess associated with the small group is $E_{B-V}=0.27 \pm 0.08$ mag and the dust distribution shows great variability across its face. We think that the small open cluster has gone undetected due to the lack of bright member stars in it.
\end{abstract}

Key words. ISM: dust, extinction - open clusters and associations: individual: NGC 5606 - techniques: polarimetric

\section{Introduction}

For some years now, we have been carrying out a Polarimetric Survey centered in open clusters of the Southern Milky Way to provide clues about the characteristics of the dust responsible for extinction and polarization in the light from member stars, to help separate between members and nonmembers, and also to study the spatial distribution of dust clouds.

As part of this survey, we present here a polarimetric investigation of NGC 5606 (C1424-594). It is a small-sized open cluster, located at galactic coordinates $l=314^{\circ} 8, b=0.9$. It consists of a central core with a size of $2 \times 2$ arcmin, where five bright stars forming a very compact group can be identified, along with a certain number of young stars surrounding that core. The first investigations were those conducted by Lyngå (1964) and Hogg (1965), followed by the works of Lyngå \& Hansson (1972) and Moffat \& Vogt (1973). More recently, two investigations

^ Based on observations obtained at Complejo Astronómico El Leoncito, operated under agreement between the Consejo Nacional de Investigaciones Científicas y Técnicas de la República Argentina and the Universities of La Plata, Córdoba, and San Juan.

$\star \star$ Table 1 is only available in electronic form at http: //www . aanda.org

$\dagger$ Member of the Carrera del Investigador Científico of CONICET. by Vázquez \& Feinstein (1991, hereafter VF91) and Vázquez et al. (1994), have contributed to deeply improve the cluster's parameters.

In particular, the last two investigations found that the cluster has about 24 members up to $V=13.5$ mag with $90 \%$ of them located inside a $10^{\prime}$ area around the central core, as well as a great number of possible members. As a by-product, VF91 identified a group of foreground late B and A type stars in the cluster's direction with a distance modulus of $V_{\mathrm{o}}-M_{v}=9.80 \pm 0.03$ (close to the near side of the Sagittarius arm). NGC 5606 is very young, as indicated by the presence of a B0.5 spectral type star still on the ZAMS. There is a slightly variable reddening across the cluster's face and the mean color excess, as found by FV91, reaches $E_{B-V}=0.51$, similar to the one derived by Moffat \& Vogt (1973): $E_{B-V}=0.49$. Based on the last studies, the distance modulus is $V_{\mathrm{o}}-M_{v}=11.90 \pm 0.20(d=2400 \mathrm{pc}$, VF91 $)$ and its age, from isochrones fitting, is between $6.3 \times 10^{6}$ and $7 \times 10^{6}$ yrs.

In previous investigations, due to the absence of proper motion studies in the cluster region, likely cluster members were identified as those stars simultaneously having reconcilable positions in color-color and color-magnitude diagrams. As some of these positions are relatively ambiguous, we decided to perform polarimetric observations of stars in the direction of the 
Table 2. Polarization results of stars in direction to NGC 5606.

\begin{tabular}{|c|c|c|c|c|c|c|c|}
\hline $\operatorname{Star}^{a}$ & $\begin{array}{c}P_{\max } \\
\%\end{array}$ & $\begin{array}{l}\epsilon_{p} \\
\%\end{array}$ & $\sigma_{1}^{b}$ & $\begin{array}{c}\lambda_{\max } \\
\mu \mathrm{m}\end{array}$ & $\begin{array}{c}\epsilon_{\lambda} \\
\mu \mathrm{m}\end{array}$ & $S p . T^{c}$ & Membership revision \\
\hline $1 *$ & 3.01 & 0.04 & 2.07 & 0.57 & 0.02 & B0 IV & nonmember \\
\hline $2^{*}$ & 3.22 & 0.29 & 2.17 & 0.50 & 0.07 & B0 IV & \\
\hline $3 *$ & 3.70 & 0.30 & 4.12 & 0.56 & 0.11 & & \\
\hline $4 *$ & 2.75 & 0.14 & 1.36 & 0.44 & 0.05 & & \\
\hline 5 & 2.69 & 0.07 & 1.95 & 0.57 & 0.03 & & \\
\hline $6^{*}$ & 3.08 & 0.14 & 1.27 & 0.56 & 0.05 & & \\
\hline $7 *$ & 3.06 & 0.37 & 1.48 & 0.54 & 0.12 & & \\
\hline $9 *$ & 4.46 & 0.33 & 0.61 & 0.70 & 0.05 & & nonmember \\
\hline $10^{*}$ & 2.41 & 0.02 & 0.44 & 0.71 & 0.01 & & \\
\hline $11 *$ & 2.82 & 0.08 & 1.07 & 0.55 & 0.04 & & \\
\hline $12 *$ & 3.45 & 0.21 & 2.50 & 0.56 & 0.09 & & \\
\hline $13 *$ & 3.69 & 0.16 & 1.44 & 0.59 & 0.04 & & \\
\hline $14 *$ & 2.23 & 0.08 & 0.35 & 0.38 & 0.02 & & prob. nonmember \\
\hline $15^{*}$ & 3.87 & 0.24 & 1.18 & 0.58 & 0.05 & B0 IV & \\
\hline $17 *$ & 3.07 & 0.12 & 1.65 & 0.59 & 0.04 & B0 IV & \\
\hline $19 *$ & 3.58 & 0.22 & 1.33 & 0.57 & 0.06 & & \\
\hline 20 & 2.94 & 0.03 & 1.13 & 0.52 & 0.01 & & new possible member \\
\hline 21 & 3.03 & 0.09 & 0.82 & 0.58 & 0.03 & & new possible member \\
\hline 24 & 1.80 & 0.21 & 1.91 & 0.62 & 0.18 & & \\
\hline 26 & 3.00 & 0.11 & 0.75 & 0.57 & 0.06 & & new possible member \\
\hline 27 & 1.83 & 0.12 & 1.05 & 0.59 & 0.09 & & in new cluster \\
\hline 28 & 1.29 & 0.06 & 0.52 & 0.57 & 0.05 & & \\
\hline $31 *$ & 2.83 & 0.43 & 1.20 & 0.41 & 0.13 & & \\
\hline 33 & 2.98 & 0.15 & 1.75 & 0.78 & 0.04 & & in new cluster \\
\hline 35 & 2.12 & 0.05 & 0.97 & 0.53 & 0.03 & & in new cluster \\
\hline $36^{*}$ & 3.17 & 0.14 & 2.23 & 0.52 & 0.05 & & \\
\hline 37 & 1.84 & 0.17 & 1.33 & 0.41 & 0.06 & & \\
\hline $38^{*}$ & 3.39 & 0.33 & 1.35 & 0.86 & 0.04 & & \\
\hline 39 & 2.15 & 0.11 & 0.75 & 0.86 & 0.06 & & in new cluster \\
\hline $40 *$ & 3.47 & 0.12 & 0.81 & 0.56 & 0.03 & & \\
\hline 41 & 2.87 & 0.17 & 2.92 & 0.69 & 0.09 & & \\
\hline 42 & 1.74 & 0.20 & 2.40 & 0.47 & 0.11 & & \\
\hline 43 & 2.16 & 0.07 & 0.68 & 0.57 & 0.04 & & in new cluster \\
\hline 44 & 2.10 & 0.12 & 1.45 & 0.62 & 0.09 & & \\
\hline 45 & 1.10 & 0.04 & 0.55 & 0.70 & 0.05 & & \\
\hline 46 & 2.28 & 0.10 & 0.71 & 0.54 & 0.06 & & \\
\hline 47 & 1.66 & 0.30 & 0.59 & 0.87 & 0.18 & & \\
\hline 48 & 2.09 & 0.15 & 1.39 & 0.54 & 0.08 & & \\
\hline 49 & 2.22 & 0.48 & 1.43 & 0.81 & 0.14 & & \\
\hline 50 & 2.34 & 0.04 & 0.40 & 0.61 & 0.03 & & in new cluster \\
\hline 52 & 2.76 & 0.42 & 2.37 & 0.34 & 0.10 & & \\
\hline 53 & 2.21 & 0.19 & 3.20 & 0.48 & 0.09 & & \\
\hline 54 & 2.37 & 0.15 & 0.68 & 0.51 & 0.08 & & in new cluster \\
\hline 55 & 2.08 & 0.13 & 1.30 & 0.64 & 0.07 & & \\
\hline 57 & 5.17 & 0.50 & 1.15 & 0.70 & 0.12 & & \\
\hline $58^{*}$ & 2.89 & 0.14 & 1.33 & 0.59 & 0.06 & & \\
\hline $59 *$ & 2.89 & 0.07 & 1.10 & 0.61 & 0.03 & & \\
\hline $60 *$ & 3.08 & 0.11 & 0.86 & 0.61 & 0.04 & & \\
\hline 61 & 3.50 & 0.31 & 1.25 & 0.76 & 0.08 & & new possible member \\
\hline 63 & 3.83 & 0.39 & 0.82 & 0.61 & 0.23 & & \\
\hline 64 & 2.01 & 0.05 & 0.93 & 0.52 & 0.04 & & in new cluster \\
\hline 65 & 1.41 & 0.04 & 1.47 & 0.55 & 0.03 & & in new cluster \\
\hline 66 & 1.20 & 0.06 & 0.83 & 0.55 & 0.07 & & in new cluster \\
\hline 68 & 1.98 & 0.25 & 1.22 & 0.62 & 0.12 & & in new cluster \\
\hline
\end{tabular}

${ }^{a}$ Identifications from Vázquez \& Feinstein (1991); * stands for member in NGC 5606 according to them. ${ }^{b} \sigma_{1}{ }^{2}=\sum\left(r_{\lambda} / \epsilon_{p_{\lambda}}\right)^{2} /(m-2)$; where $m$ is the number of colors and $r_{\lambda}=P_{\lambda}-P_{\max } \exp \left(-K \ln ^{2}\left(\lambda_{\max } / \lambda\right)\right) .{ }^{c}$ Spectral type from the WEBDA database.

open cluster in order to clarify memberships, to study the particular characteristics of the dust between cluster and Sun and, finally, to determine if the group of stars identified in VF91 has any physical entity as their photometric plots suggest.

\section{Observations}

Observations in the $U B V(R I)_{\mathrm{KC}}$ bands (KC: Kron-Cousins, $\lambda_{U_{\mathrm{eff}}}=0.36 \mu \mathrm{m}, F W H M=0.05 \mu \mathrm{m} ; \lambda_{B_{\mathrm{eff}}}=0.44 \mu \mathrm{m}, F W H M=$ $0.06 \mu \mathrm{m} ; \lambda_{V_{\text {eff }}}=0.53 \mu \mathrm{m}, F W H M=0.06 \mu \mathrm{m} ; \lambda_{R_{\mathrm{eff}}}=0.69 \mu \mathrm{m}$, 


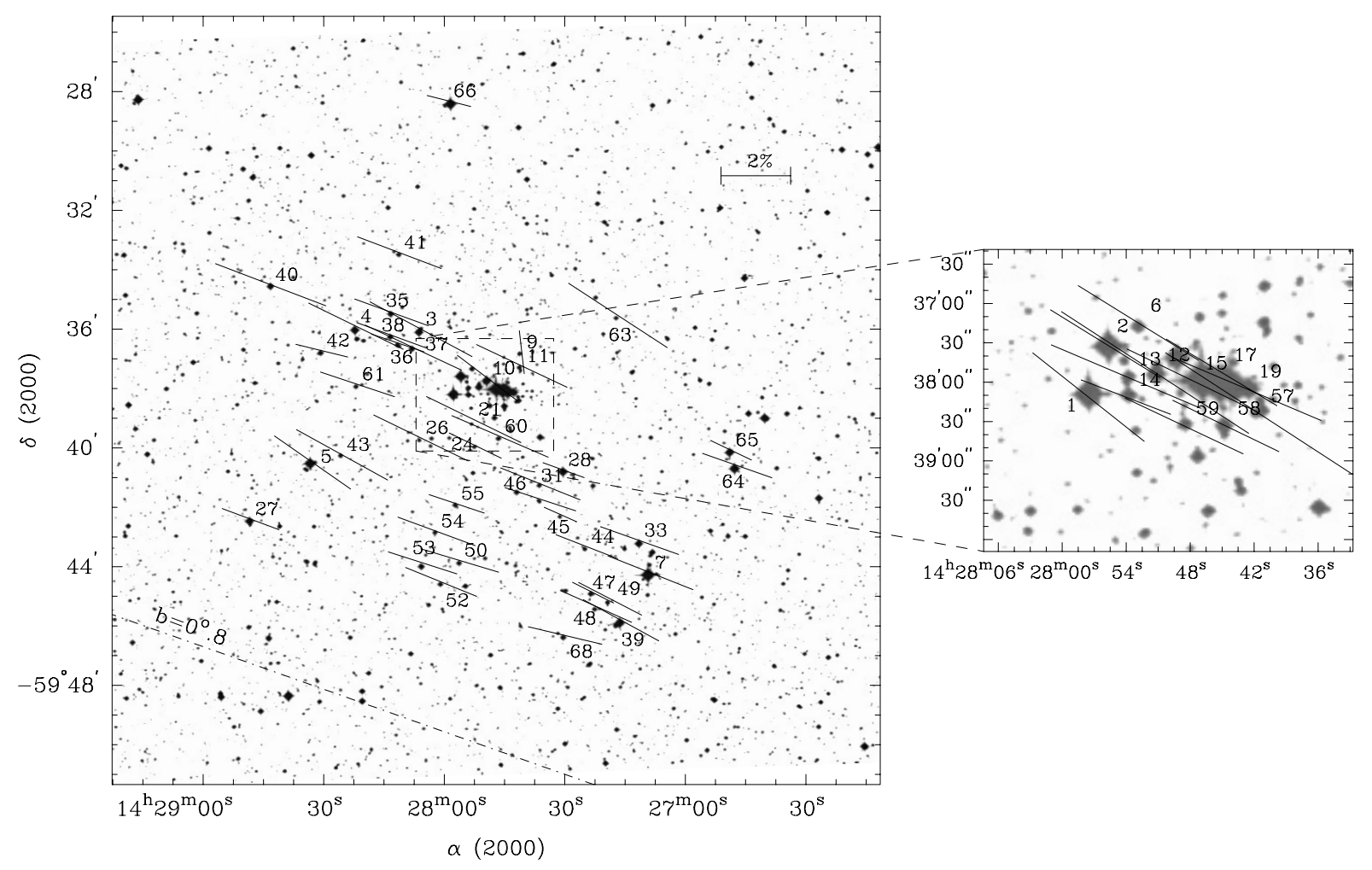

\section{NGC 5606}

Fig. 1. Projection on the sky of the polarization vectors (Johnson $V$ filter) of the stars observed in the region of NGC 5606. The dot-dashed line is the galactic parallel $b=0.8$. The lengh of each vector is proportional to the percentage polarization.

$\left.F W H M=0.18 \mu \mathrm{m} ; \lambda_{I_{\text {eff }}}=0.83 \mu \mathrm{m}, F W H M=0.15 \mu \mathrm{m}\right)$ were carried out using the five-channel photopolarimeter of the Torino Astronomical Observatory attached to the $2.15 \mathrm{~m}$ telescope at the Complejo Astronómico El Leoncito (San Juan, Argentina). They were performed on six nights (April 6-9; May 7-8) in 2005. Standard stars for null polarization and for the zero point of the polarization position angle were taken from Clocchiatti \& Marraco (1988). For further information on the instrument, data acquisition and data reduction, see Scaltriti (1994).

Table 1 lists the 54 stars observed polarimetrically in the direction of the open cluster, the percentage polarization $\left(P_{\lambda}\right)$, the position angle of the electric vector $\left(\theta_{\lambda}\right)$ in the equatorial coordinate system, and their respective mean errors for each filter. We also indicate the number of 60-s integrations with each filter. Star identifications are taken from VF91, but as noted in Vázquez et al. (1994), we have swapped the ids of stars 13 and 14. Possible members according to these authors ( 22 stars), are identified by an asterisk in both Tables 1 and 2. The spectroscopic data were obtained from the WEBDA (http://obswww. unige.ch/webda/) Open Cluster Database of Mermilliod (1988, 1992).

\section{Results}

The sky projection of the $V$-band polarization vectors for the observed stars in the direction of NGC 5606 are shown in Fig. 1. The dot-dashed line superimposed on the figure is the Galactic parallel $b=0.8$, denoting an alignment of the polarization vectors with the projection of the Galactic Plane.

This indicates that the dust grains along the line of sight are aligned by a magnetic field which is also close to the direction of the Galactic Disk, implying that the dust layers responsible for the observed polarization are located in a rather undisturbed place in our Galaxy.

Figure 2 (upper plot) displays the relation that exist between $P_{V}$ and the position angle of the polarization vector $\theta_{V}$, where filled circles and open triangles are used for members and nonmembers, respectively. According to this figure, members have higher polarizations than nonmembers, since there is no single member with a polarization $P_{V}<2 \%$. Also, there is a notable concentration of members with a $\theta_{V}$ of $64^{\circ}$ and $P_{V} \sim 3 \%$, and some spread of data showing lower angles. The nonmember stars show a different point of concentration, near $\theta_{V}=69^{\circ}$ and $P_{V}=2 \%$. This angle, as it is shown in the plot, is the orientation of the Galactic Disk.

Figure 2 (middle plot) displays a histogram of the observed angles of the polarization vector for the nonmember stars as classified by VF91. The plot shows that these stars have a noticeable large spread, in a range from $55^{\circ}$ to $78^{\circ}$. Also the histogram has the maximum on the projection of the orientation of Galactic Disk over the region. Meanwhile Fig. 2 (lower plot) is the same plot, but for the considered member stars (VF91). Notice that this plot shows almost a lack of members with angles higher than $70^{\circ}$ (close to Galactic Disk). So, the members diplay a polarization angle which is somewhat different from that of the 


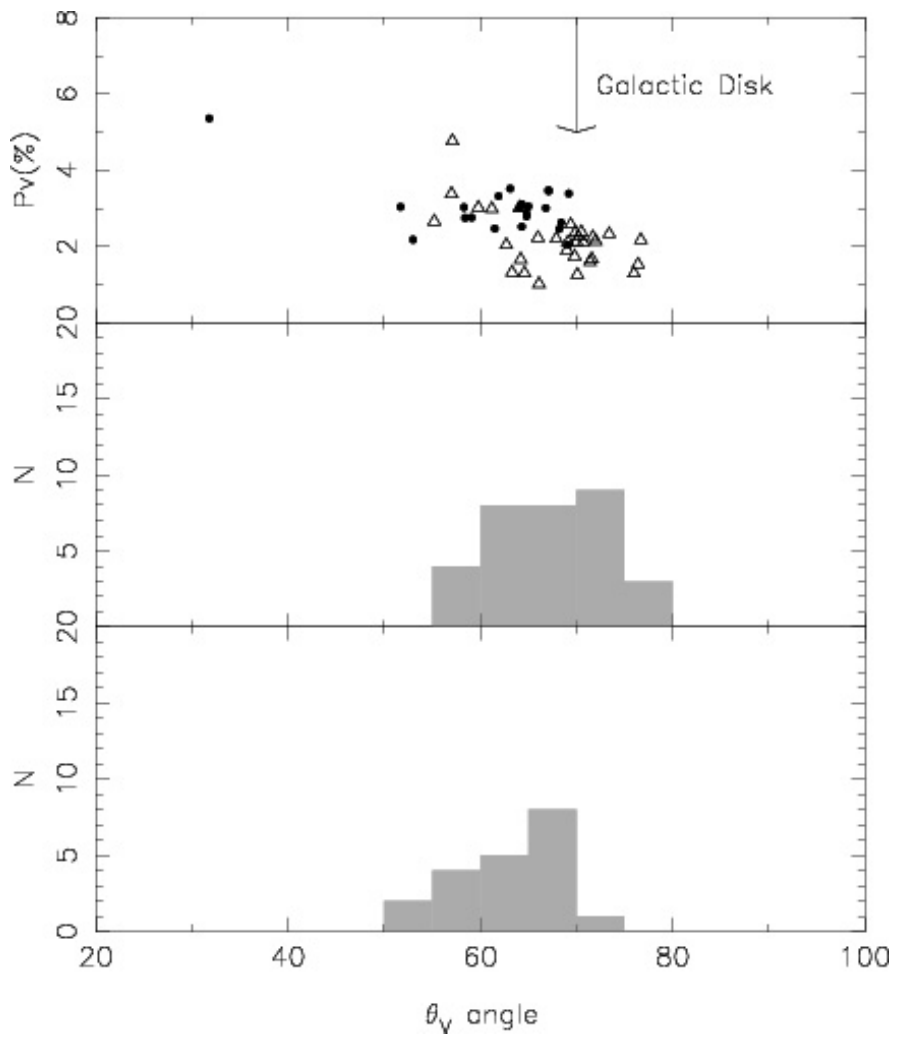

Fig. 2. Upper plot: $V$ band polarization percentage of the stellar flux $P_{V}(\%)$ vs. the polarization angle $\theta_{V}$ for each star. Filled circles are used for member stars, while open triangles are for nonmembers, from the work of Vázquez \& Feinstein (1991). Middle plot: histogram of the polarization angle $\left(\theta_{V}\right)$ for observed stars considered nonmembers. Lower plot: histogram of the polarization angle $\left(\theta_{V}\right)$ for the stars considered members. The arrow displays the location of the projection of the Galactic Plane in the region. Note that most of the member stars are located at angles lower than $70^{\circ}$, so at lower angles than the projection of the Galactic Disk in the region.

nonmembers (and the Galactic Disk). Also the spread in the polarization angle is larger than the average observed for a cluster in other investigations, which are commonly less than $10^{\circ}$, as found, e.g., in NGC 6193, NGC 6167, NGC 6204, Hogg 22, Trumpler 27, Stock 16 (Waldhausen 1999; Martínez et al. 2004; Feinstein et al. 2000; Feinstein et al. 2003).

These two histograms could indicate a contamination of the sample with some stars wrongly classified as members, although an Kolmogorov-Smirnow test shows that these distributions have a low probability of being different only by chance $(11 \%)$. In the following sections we are going to test the stars membership throughout polarimetric data.

\section{Analysis and discussion}

\subsection{The fitting with the Serkowski law}

The polarimetric observations in the five filters were fitted in each star using Serkowski's law of interstellar polarization (Serkowski 1973). That is:

$P_{\lambda} / P_{\lambda \max }=\mathrm{e}^{-K \ln ^{2}\left(\lambda_{\max } / \lambda\right)}$.

We assume that if polarization is produced by aligned interstellar dust particles, then the observed data (in terms of wavelength,
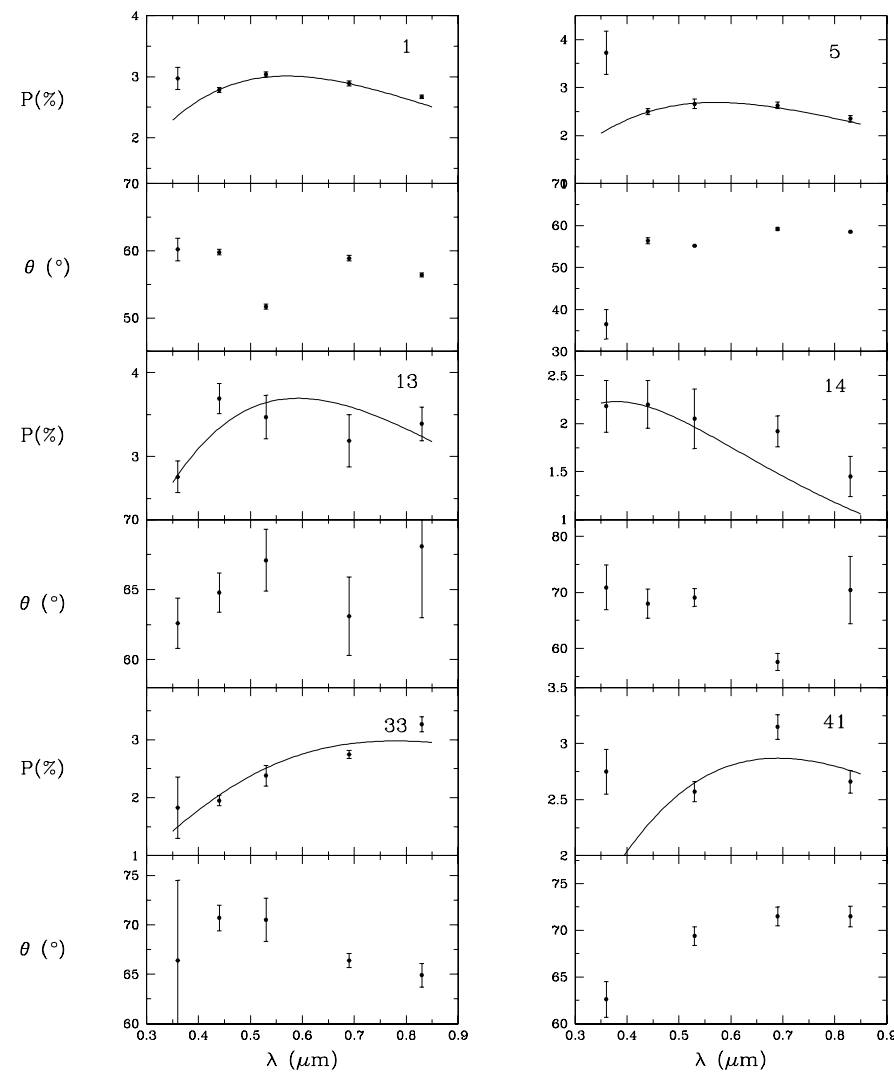

Fig. 3. The plot displays both the polarization and position angle dependence on wavelength, for stars with indications of intrinsic polarization. The numbers in the polarization dependence plots are the star id. and right below is the position angle dependence for each star.

UBVRI) will follow (1) and each star will have a $\lambda_{\max }$ and a $P_{\lambda_{\max }}$ values.

To perform the fitting we have adopted $K=1.66 \lambda_{\max }+0.01$, with $\lambda_{\max }$ in $\mu \mathrm{m}$ (Whittet et al. 1992). For each star, we have also computed the $\sigma_{1}$ parameter (the unit weight error of the fit) in order to quantify the departure of our data from the "theoretical curve" of Serkowski's law. In our scheme, when a star shows $\sigma_{1}>1.5$, it is indicating the presence of intrinsic stellar polarization. The dominant source of intrinsic polarization is dust distributed non-spherically and, for classical Be stars, electron scattering. The $\lambda_{\max }$ values can also be used to test the origin of the polarization: those objects having a $\lambda_{\max }$ much shorter than the average value of the interstellar medium $(0.55 \mu \mathrm{m}$, Serkowski et al. 1975) are candidates for having an intrinsic component of polarization as well (cf. Orsatti et al. 1998). The individual $P_{\lambda_{\max }}, \sigma_{1}, \lambda_{\max }$, and $\bar{\epsilon}$ values, together with the star identifications from VF91 are listed in Table 2. The mathematical expression used to obtain the individual $\sigma_{1}$ values is found in this table as a footnote.

Figure 3 shows the observed $P_{\lambda}$ and $\theta_{\lambda}$ vs. $\lambda$ for some stars which are likely to have an intrinsic component of polarization, from a total number of 17 out of the 54 observed stars. For comparison purposes, the best Serkowski-law fit has been plotted as a continuous line.

On possible origins of the intrinsic polarization in the light of these stars, we have found that \#1 (CPD -59 5580 = LS 3243, member according to VF91 (1991)) is an evolved object (BO IV; FizGerald et al. 1979). Using Schmidt-Kaler's calibration, we get a distance of $3698 \mathrm{kpc}$ from the Sun, which locates the star in the background and not as the same distance as the cluster: 


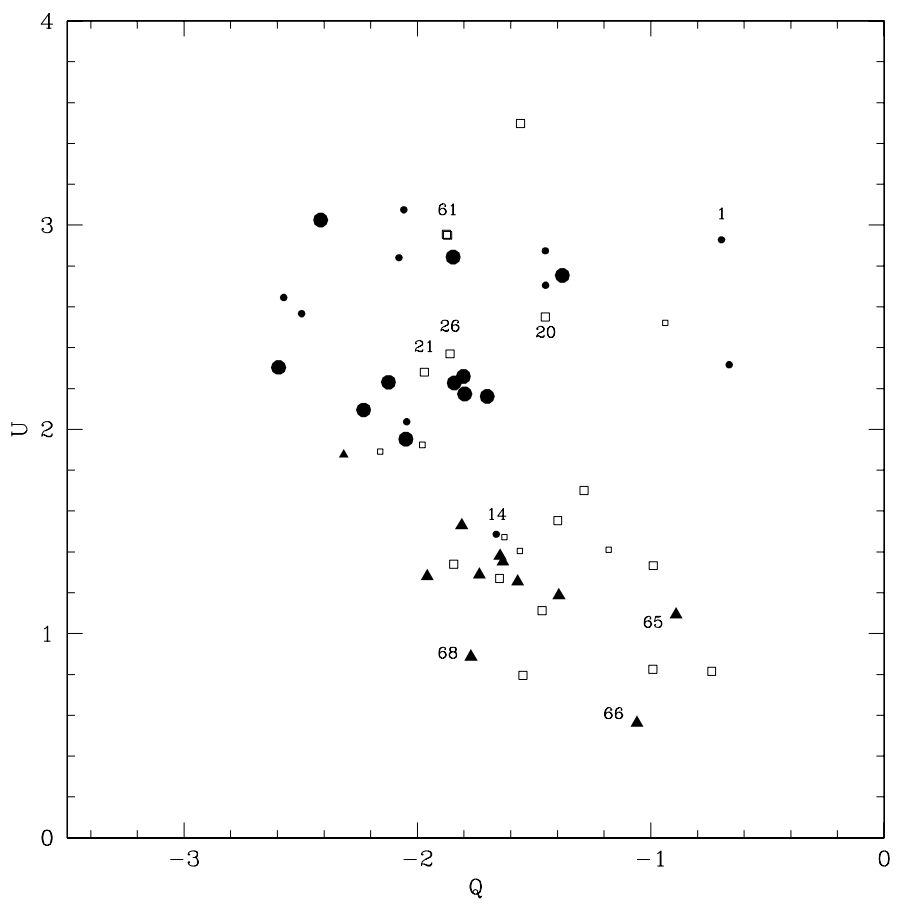

Fig. 4. $Q$ - and $U$-parameters for the $V$ bandpass. Circles are for members of NGC 5606, triangles are for stars of the new open cluster, and squares are the observed stars which are not part of either cluster. Small symbols are used for stars with intrinsic polarization.

2400 pc. It has a $P_{\max }$ higher than the mean for cluster members (see Sect. 4.2) and a $\theta_{v}$ value also different when compared to the corresponding mean for the object. As a conclusion, we consider \#1 as a background star and not a member of the open cluster.

Star \#5 (nonmember, HD 126577) has an IRAS identification (14248-5927). Kwok et al. (1997) classified this M2/M3 star as of type E, that is: "an M star whose spectra shows a silicate dust feature in emission (at $9.7 \mu \mathrm{m}$ ), originated in a circumstellar envelope". The $P_{\lambda}$ vs. $\lambda$ plot shows that all the filters fit the Serkowski relation well, except in the case of the $U$ bandpass, with the $P_{U}$ measure near the 2-sigma of the fit.

In the $\theta_{\lambda}$ vs. $\lambda$ plot for the star, the $\theta_{U}$ does not follow the horizontal trend of the rest of the angles and the measurement is well outside the 2-sigma of the fit. This situation, plus Kwok et al. 1997's classification could indicate the presence of intrinsic polarization. We will refer to star \#14 later in the following subsection.

\subsection{The $Q$ vs. $U$ plot}

The plot of the Stokes parameters $Q$ vs. $U$ for the $V$ bandpass, where $Q=P_{v} \cos \left(2 \theta_{v}\right)$ and $U=P_{v} \operatorname{sen}\left(2 \theta_{v}\right)$, is shown in Fig. 4. They are the components, in the equatorial system, of the polarization vector $P_{v}$. This plot supplies useful information on variations in interstellar environments: if the light from individual members of an open cluster has gone through a common sheet of dust, their representative points will concentrate on a given region of the plot, indicating similar optical characteristics of the ISM. If the plot shows more than a concentration, this means that the light from a certain group of stars has traveled through different regions of dust with somewhat different characteristics.

In this figure we confirm the result found in the previous section, about the existence of two different groupings: one of them

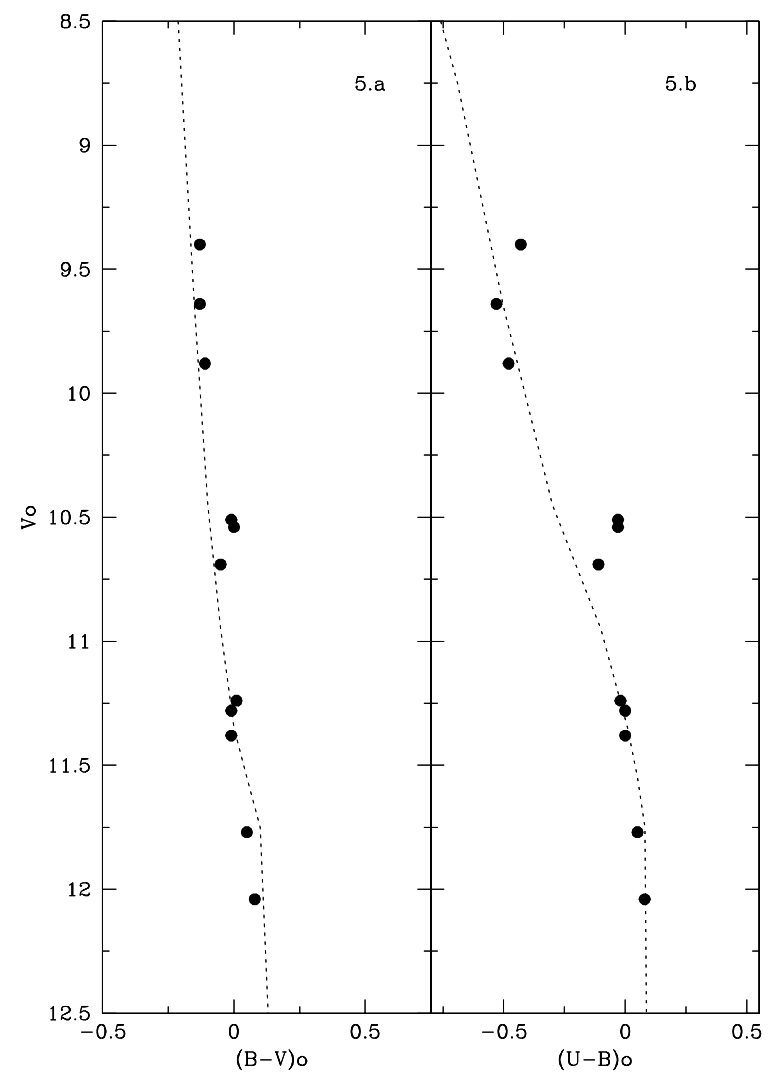

Fig. 5. $V_{\mathrm{o}}$ vs. $(B-V)_{\mathrm{o}}$ a) and $V_{\mathrm{o}}$ vs. $(U-B)_{\mathrm{o}}$ b) diagrams for the possible cluster members. The Schmidt-Kaler's ZAMS (1982) is shown as fitted to a distance modulus $V_{\mathrm{o}}-M_{v}=9.90$. Star 14 has not been included.

(upper group) composed mainly of stars listed by VF91 as members (filled circles), and a second one (lower group) with stars accepted by them as nonmembers of NGC 5606 (these, shown with triangles and squares, whose meaning will be clarified in the next subsection). We have mentioned before that the value of the parameter $\sigma_{1}$ (the unit weight error of the fit) should not be higher than 1.5 if the measured polarization has an interstellar origin. If this is not the case, then we have detected the presence of intrinsic polarization in the light from the star, i.e., a polarization that did not originate in the ISM between the star and the Sun. Small symbols in Fig. 4 are used to indicate these stars with intrinsic polarization.

Stars \#9 and 57 (member and nonmember respectively, both according to VF91) fall outside the plot limits; for \#9, in particular, it indicates an undetected intrinsic polarization or that the star is not member of NGC 5606. We think this is the correct answer since the corresponding $\mathrm{Q}$ and $\mathrm{U}$ for the star are very different compared to the rest of the members. The dispersion of the points for NGC 5606 in this Fig. 4 indicates some slightly different polarimetric characteristics in the dust located between the stars and the Sun. This could be due to the existence of intracluster dust, not really unusual if we think about the young age of the cluster. Stars \#20, 21, 26, and 61 (no photometric observations) are plotted in the region of the cluster stars. We think they are new members. To derive mean values for maximum polarization and polarization angle, we use the seven stars with similar Stokes parameters: \#4, \#11, \#58, \#59 and the new members $\# 20$, \#21, and \#26. In the cluster chart, they are seen near the central concentration. We obtain $P_{\max }=2.97$ and $\theta_{v}=63.5$ (mean of 7); if we use the 15 members, we get values for $P_{\max }$ 


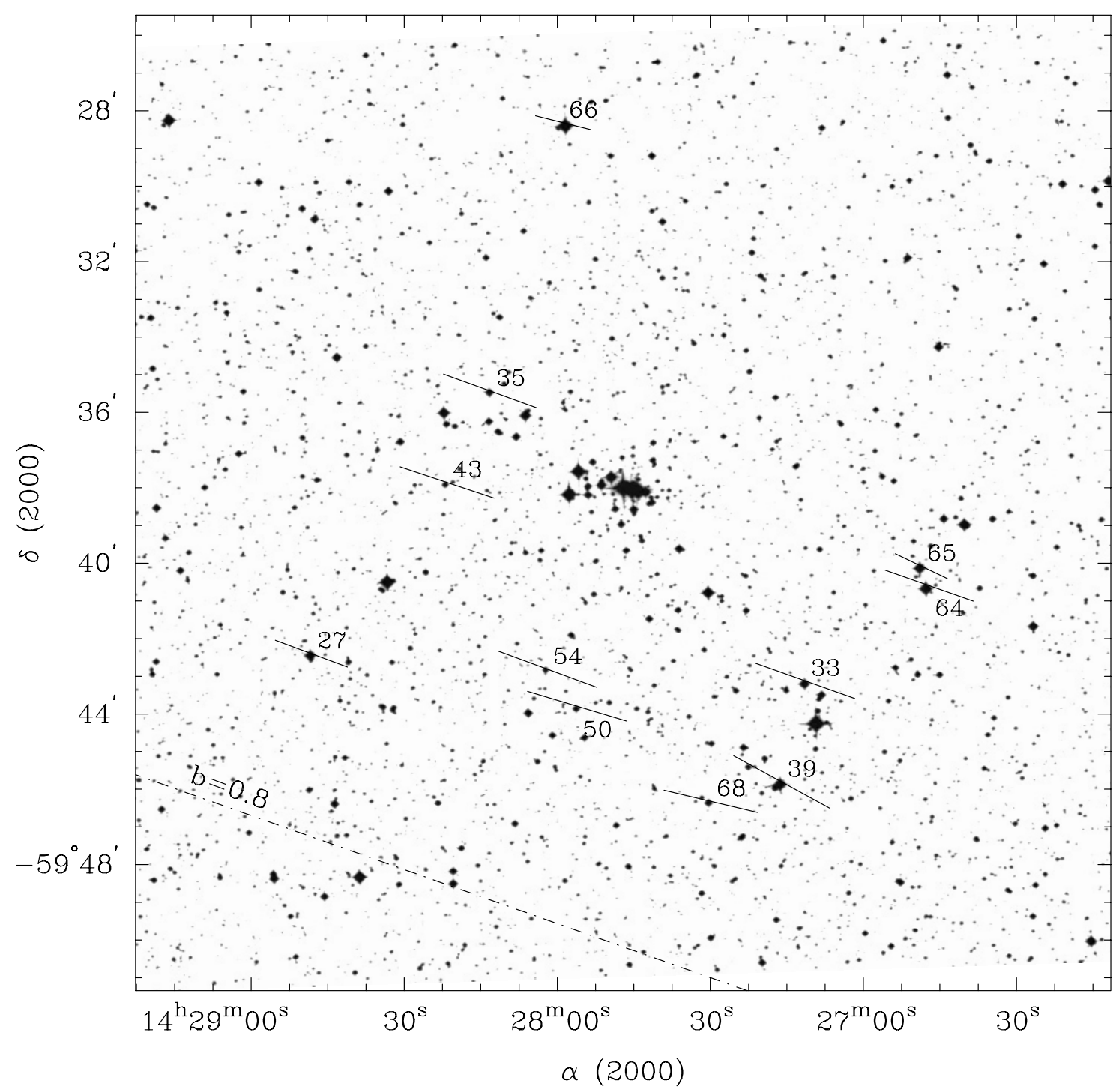

Fig. 6. Anon, (La Plata 1) spatial distribution across the face of NGC 5606.

and $\theta_{v}$ of 3.08 and $64^{\circ} 3$ respectively. The mean $\lambda_{\max }$ amounts to $0.55 \pm 0.06 \mu$, the value associated to the ISM.

\subsection{A possible new open cluster}

The lower group in Fig. 4 includes the 11 late B- and A-spectral type stars identified by VF91 as foreground. VF91 did not go further analyzing a possible physical characterization of these stars, as would suggest the small error in mean distance $( \pm 0.3)$. To investigate if the stars had anything in common, we reproduced the VF91's photometric plots $V_{\mathrm{o}}$ vs. $(B-V)_{\mathrm{o}}$ and $V_{\mathrm{o}}$ vs. $(U-B)_{\mathrm{o}}$ (Figs. 5a and b, respectively) only for the eleven stars, avoiding in this way the visual confusion introduced by the rest of the observations in the region. The original VF91's observations were individually dereddened along reddening lines, obtaining intrinsic colors $(B-V)_{\mathrm{o}}$ and $(U-B)_{\mathrm{o}}$. The fitting procedure of Schmidt-Kaler's ZAMS (1982) was used to derive the distance to the group by fitting the ZAMS in the $V_{\mathrm{o}}$ vs. $(U-B)_{\mathrm{o}}$ plot and obtaining a distance modulus of $V_{\mathrm{o}}-M_{v}=9.90$ (very similar to that of VF91), with a mean color excess associated of $E_{B-V}=0.27 \pm 0.08 \mathrm{mag}$; the dispersion is due to great variability of the dust distribution across the face of the cluster. Using these values, we have calculated a distance of $649 \mathrm{pc}$ from the Sun. We want to emphasize that no new photometric observations were used here other than those from the work of VF91.

The new cluster, which we have provisionally named Anon (La Plata 1), extends from NE to SW across the face of NGC 5606 (Fig. 6) covering about 10' which, at the cluster's distance, gives a few pc in diameter. It has characteristics of an intermediate age open cluster, due to the presence of a star with a photometric spectral type b6 star, still on the ZAMS. Using Table 2 in Meynet et al. (1993), we get an approximate age of turnoff $(B-V)$ between 0.79 and $1 . \times 10^{8}$ yrs.

To get mean polarimetric parameters for the interstellar medium associated with the new open cluster, we selected 7 out of 11 members, discarding \#33, 65, 66, and 68. The first star has indications of intrinsic polarization; and the light from the other four has traveled through dust clouds of different polarimetric characteristics since for them we get lower polarization values than for the rest of La Plata 1 member stars. We have obtained a mean $P_{\max }$ and $\theta_{v}$ values of $2.12 \pm 0.27$ and 70.9, respectively, and the $\lambda_{\max }$ is similar to that for the normal ISM: $0.55 \pm 0.03$.

We searched for other members in VF91's Table 1. But unfortunately, for visual magnitudes fainter than $V=12.5 \mathrm{mag}$, the 


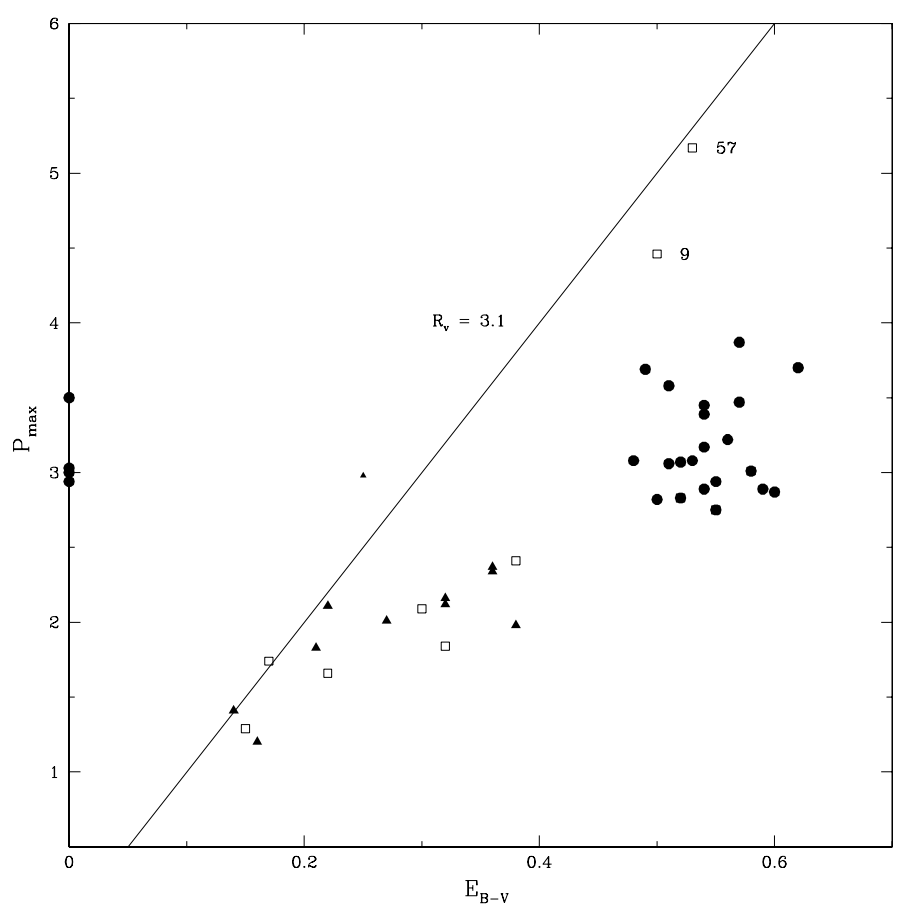

Fig. 7. Polarization efficiency diagram. Symbols are the same as in Fig. 4. The background stars \#9 and \#57 are also indicated. The line of maximum efficiency is drawn adopting $R_{v}=3.1$.

$U B V$ photoelectric observations made by the authors with the $60 \mathrm{~cm}$-Lowell telescope at CTIO seem to be clearly affected by observational errors, due to the small aperture of the telescope in use or to weather observational conditions. Also, as the CCD work of Vázquez et al. (1994) is so concentrated on the central region of NGC 5606, there is no coverage at all of the surroundings. For that reason we have not been able to improve the cluster stellar content. The only exception could be star \#14 (member of NGC 5606 according to VF91). Its location in the $Q$ vs. $U$ plot (Fig. 4) falls on the front-side stars group, indicating that it does not share similar polarimetric characteristics with the rest of the NGC 5606 cluster members. It has a $P_{\max }=2.23 \pm 0.08 \%$ and $\theta_{V}=69^{\circ} .1$, both values very similar to those found for stars in La Plata 1. The $\lambda_{\max }$ for this star amounts to $0.38 \pm 0.02$, indicating intrinsic polarization in the light. For that reason, the membership of this star in the new cluster cannot be confirmed. We conclude that the group could be physically related and may be considered as a possible new open cluster, which went undetected due to the lack of bright member stars in it.

\subsection{Polarization efficiency}

It is known that for the interstellar medium the polarization efficiency (ratio of the maximum amount of polarization to visual extinction) rarely exceeds the empirical upper limit,

$P_{\max } \leq 3 A_{v} \simeq 3 R_{v} E_{B-V}$

obtained for interstellar dust particles (Hiltner 1956). The ratio $P_{\max } / R_{v} \cdot E_{B-V}$ depends mainly on the alignment efficiency and the magnetic field strength, and also on the amount of depolarization due to radiation traversing more than one cloud with different field directions.

Figure 7 depicts the relation that exists between reddening and polarization originated in dust along the line of sight to
NGC 5606. Excesses $E_{B-V}$ were obtained from the literature or from the relationship between spectral type and color indexes following Schmidt-Kaler (1982). It can be seen that except for one star (\#33), the rest lie on (or to the right of) the interstellar maximum line, indicating that the observed polarization is mostly due to the ISM. Star 9, mentioned before as plotted outside the $Q$ vs. $U$ plot, shows in this figure characteristics of a nonmember star; for that reason, we decided to plot it as a background star.

This figure also shows that while the excesses for NGC 5606's members run from 0.46 to 0.62 mag approximately, the variation in polarization values is high: about $1 \%$. We think that this high variation in $P_{\max }$ indicates the presence of intracluster dust.

Not all of the observed stars could be plotted in this figure due to the lack of calculated color excesses as is the case for $\# 20,21,26$, and 61, new possible members of NGC 5606. We decided to indicate their positions along the $P_{\max }$ axis to show that their polarization values are similar to those associated with cluster stars.

\section{Summary}

We have observed linear multicolor polarization for a sample of 54 stars on the region of the open cluster NGC 5606. Through the analysis of the polarimetric data, we have found at least two dust layers along the line of sight to this open cluster. The observations show that both dust layers have their local magnetic field with an orientation which is close to the direction of the Galactic Plane $\left(\theta=70^{\circ} .0\right)$, but the direction of the polarimetric vector for the members of the cluster seems to be lower than this value.

Although showing some dispersion, NGC 5606 can be polarimetrically characterized with $P_{\max }=2.79 \%$ and $\theta_{v}=63.5$. The spread in polarization values for the members of NGC 5606 seems to be compatible with intracluster dust. We have confirmed 18 memberships in NGC 5606, except for stars \#1, 9, and \#14; and we have found that four other stars (\#20, 21, 26, and 61) qualify for membership in this cluster, through their positions in the $Q$ vs. $U$ polarimetric plot. The polarimetric techniques have helped to separate between front-side and background stars between those observed in direction to the open cluster, as summarized in the last column of Table 2 .

Some of the background stars show very similar characteristics in polarization and $\theta_{v}$ values. We believe that these stars are part of a new and undetected open cluster since the HR diagrams, using previous photometric measurements from VF91, show a noticeable main sequence. This new object has an extinction of $E_{B-V}=0.27 \pm 0.08$ mag and a probable age between 0.79 and $1 . \times 10^{8} \mathrm{yr}$. The number of members may be far from complete, since VF91 planned the photometry in the region so as to fulfill another investigation.

Acknowledgements. We wish to acknowledge the technical support and hospitality at CASLEO during the observing runs. We also acknowledge the use of the Torino Photopolarimeter built at Osservatorio Astronomico di Torino (Italy) and operated under agreement between Complejo Astronómico El Leoncito and Osservatorio Astronomico di Torino.

Special thanks go to Mrs. M. C. Fanjul de Correbo and Mr. Ruben Martínez for technical assistance.

\section{References}

Clocchiatti, A., \& Marraco, H. G. 1988, A\&A, 197, L1

FitzGerald, M. P., Luiken, M., Maitzen, H. M., \& Moffat, A. F. J. 1979, A\&AS, 37,345 
Feinstein, C., Baume, G., Vázquez, R., Niemela, V., \& Cerruti, M. A. 2000, AJ, 120, 1906

Feinstein, C., Baume, G., Vergne, M. M., \& Vázquez, R. 2003, A\&A, 409, 933 Hiltner, W. A. 1956, ApJS, 2, 389

Hogg, A. R. 1965, Catalogue of Open South Cluster of $-45^{\circ}$ Declination, Australian National University, Camberra

Kwok, S., Volk, K., \& Bidelman, W. P. 1997, ApJS, 112, 557

Lyngå, G. 1964, Medd. Lunds Astron Obs. Ser., 6, 237

Lyngå, G., \& Hansson, N. 1972, A\&AS, 6, 327

Martínez, R., Vergne, M. M., \& Feinstein, C. 2004, A\&A, 419, 965

Mermilliod, J.-C. 1988, Bull. Inform. CDS, 35, 77

Mermilliod, J.-C. 1992, Bull. Inform. CDS, 40, 115

Meynet, G., Mermilliod, J.-C., \& Maeder, A. 1993, A\&A, 98, 477
Moffat, A. F. J., \& Vogt, N. 1973, A\&AS, 10, 135

Orsatti, A. M., Vega, E. I., \& Marraco, H. G. 1998, AJ, 116, 226

Scaltriti, F. 1994, Technical Publication No TP-001, Osservatorio Astronomico di Torino

Schmidt-Kaler, Th. 1982, in Landolt/Bornstein, Neue Series VI/2b

Serkoswki, K. 1973, in Interstellar Dust and Related Topics, ed. J. M. Greenberg, \& H. C. van der Hulst (Dordrecht-Holland: Reidel), IAU Symp., 52, 145

Serkowski, K., Mathewson, D. L., \& Ford, V. L. 1975, ApJ, 196, 261

Vázquez, R. A., \& Feinstein, A. 1991, A\&AS, 87, 383

Vázquez, R. A., Baume, G., Feinstein, A., \& Prado, P. 1994, A\&A, 106, 339

Waldhausen, S., Martínez, R., \& Feinstein, C. 1999, AJ, 117, 2882

Whittet, D. C. B., Martin, P. G., Hough, J. H., et al. 1992, ApJ, 386, 562 
A. M. Orsatti et al.: Revisiting the region of the open cluster NGC 5606, Online Material p 1

\section{Online Material}


A. M. Orsatti et al.: Revisiting the region of the open cluster NGC 5606, Online Material p 2

Table 1. Polarimetric observations in direction to NGC 5606.

\begin{tabular}{|c|c|c|c|c|c|c|c|c|c|c|c|c|c|c|c|}
\hline$\overline{\text { Star }^{a}}$ & Other id. ${ }^{b}$ & Filter & $\begin{array}{l}P_{\lambda} \\
\%\end{array}$ & $\epsilon_{p}$ & $\overline{\theta_{\lambda}}$ & $\epsilon_{\theta}$ & 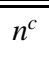 & $\overline{\text { Star }^{a}}$ & Other id. ${ }^{b}$ & Filter & $\begin{array}{l}P_{\lambda} \\
\%\end{array}$ & $\epsilon_{p}$ & $\overline{\theta_{\lambda}}$ & $\epsilon_{\theta}$ & $\overline{n^{c}}$ \\
\hline \multirow[t]{5}{*}{$\overline{\mathrm{Vz}} 1^{*}$} & MV3 & $\bar{U}$ & 2.97 & 0.18 & 60.2 & 1.7 & 2 & Vz 14* & MV7 & $\bar{U}$ & 2.18 & 0.27 & 70.9 & 4.0 & 2 \\
\hline & & $B$ & 2.78 & 0.04 & 59.8 & 0.4 & & & & $B$ & 2.20 & 0.25 & 68.0 & 2.6 & \\
\hline & & $V$ & 3.04 & 0.04 & 51.7 & 0.4 & & & & V & 2.05 & 0.31 & 69.1 & 1.6 & \\
\hline & & $R$ & 2.89 & 0.04 & 58.9 & 0.4 & & & & $R$ & 1.92 & 0.16 & 57.6 & 1.5 & \\
\hline & & $I$ & 2.67 & 0.03 & 56.4 & 0.3 & & & & $I$ & 1.45 & 0.21 & 70.4 & 6.0 & \\
\hline \multirow[t]{5}{*}{$\mathrm{Vz} 2 *$} & MV4 & $U$ & 3.65 & 0.37 & 63.4 & 2.5 & 4 & Vz $15^{*}$ & MV1 & $U$ & 3.15 & 0.16 & 64.4 & 1.7 & 4 \\
\hline & & $B$ & 3.78 & 0.39 & 58.1 & 0.7 & & & & $B$ & 3.94 & 0.42 & 63.9 & 2.6 & \\
\hline & & $V$ & 2.75 & 0.17 & 58.4 & 0.6 & & & & V & 3.11 & 0.41 & 64.3 & 2.1 & \\
\hline & & $R$ & 3.19 & 0.20 & 57.9 & 0.8 & & & & $R$ & 3.98 & 0.52 & 61.2 & 1.7 & \\
\hline & & $I$ & 2.62 & 0.13 & 58.6 & 1.4 & & & & $I$ & 3.61 & 0.41 & 65.1 & 3.2 & \\
\hline \multirow[t]{5}{*}{ Vz $3 *$} & & $U$ & 2.85 & 0.21 & 58.1 & 2.9 & 2 & Vz 17* & MV2 & $U$ & 2.61 & 0.14 & 62.4 & 0.2 & 2 \\
\hline & & $B$ & 4.87 & 0.22 & 60.5 & 1.3 & & & & $B$ & 2.81 & 0.14 & 60.3 & 1.2 & \\
\hline & & $V$ & 3.32 & 0.12 & 61.9 & 1.1 & & & & V & 2.76 & 0.10 & 59.1 & 1.2 & \\
\hline & & $R$ & 3.69 & 0.15 & 58.7 & 1.2 & & & & $R$ & 3.29 & 0.13 & 60.5 & 1.1 & \\
\hline & & $I$ & 3.48 & 0.28 & 65.0 & 2.3 & & & & $I$ & 2.74 & 0.10 & 57.2 & 1.1 & \\
\hline \multirow[t]{5}{*}{ Vz $4 *$} & & $U$ & 2.44 & 0.19 & 65.4 & 2.3 & 2 & Vz 19* & MV14 & $U$ & 2.75 & 0.20 & 62.6 & 1.9 & 3 \\
\hline & & $B$ & 2.91 & 0.17 & 63.3 & 4.0 & & & & $B$ & 3.68 & 0.18 & 64.8 & 1.4 & \\
\hline & & $V$ & 3.02 & 0.21 & 64.1 & 2.0 & & & & V & 3.46 & 0.27 & 67.1 & 2.2 & \\
\hline & & $R$ & 2.27 & 0.20 & 62.2 & 2.1 & & & & $R$ & 3.19 & 0.31 & 63.1 & 2.8 & \\
\hline & & $I$ & 2.01 & 0.23 & 67.9 & 2.4 & & & & $I$ & 3.39 & 0.61 & 68.1 & 5.1 & \\
\hline \multirow[t]{5}{*}{ Vz 5} & & $U$ & 3.73 & 0.45 & 36.5 & 3.5 & 2 & Vz 20 & MV10 & $U$ & 2.55 & 0.08 & 61.5 & 0.9 & 4 \\
\hline & & $B$ & 2.50 & 0.06 & 56.4 & 0.7 & & & & $B$ & 2.84 & 0.04 & 59.5 & 0.4 & \\
\hline & & $V$ & 2.66 & 0.10 & 55.2 & 0.1 & & & & V & 3.02 & 0.05 & 59.8 & 0.5 & \\
\hline & & $R$ & 2.63 & 0.07 & 59.2 & 0.3 & & & & $R$ & 2.82 & 0.04 & 59.6 & 0.4 & \\
\hline & & $I$ & 2.35 & 0.07 & 58.5 & 0.2 & & & & $I$ & 2.40 & 0.04 & 58.6 & 0.5 & \\
\hline \multirow[t]{5}{*}{ Vz $6^{*}$} & MV9 & $U$ & 2.33 & 0.27 & 55.7 & 3.3 & 2 & Vz 21 & & $U$ & 2.44 & 0.19 & 65.4 & 2.3 & 2 \\
\hline & & $B$ & 3.55 & 0.32 & 56.0 & 2.4 & & & & $B$ & 2.91 & 0.41 & 63.3 & 4.0 & \\
\hline & & $V$ & 3.03 & 0.21 & 58.3 & 1.2 & & & & V & 3.02 & 0.21 & 64.1 & 2.0 & \\
\hline & & $R$ & 3.03 & 0.20 & 57.6 & 1.4 & & & & $R$ & 2.76 & 0.20 & 62.2 & 2.1 & \\
\hline & & $I$ & 2.55 & 0.28 & 56.2 & 3.1 & & & & $I$ & 2.88 & 0.24 & 67.9 & 2.4 & \\
\hline \multirow[t]{5}{*}{ Vz $7 *$} & & $U$ & 2.90 & 0.44 & 69.5 & 4.4 & 2 & Vz 24 & & $U$ & 2.82 & 0.54 & 81.4 & 5.4 & 2 \\
\hline & & $B$ & 3.32 & 0.30 & 65.5 & 2.6 & & & & $B$ & 1.06 & 0.47 & 53.3 & 12.0 & \\
\hline & & $V$ & 2.63 & 0.27 & 68.4 & 2.9 & & & & $V$ & 1.66 & 0.17 & 64.2 & 3.0 & \\
\hline & & $R$ & 2.89 & 0.23 & 61.1 & 2.3 & & & & $R$ & 1.74 & 0.17 & 67.2 & 2.9 & \\
\hline & & $I$ & 1.53 & 0.50 & 56.2 & 9.0 & & & & $I$ & 2.07 & 0.34 & 80.1 & 4.7 & \\
\hline \multirow{5}{*}{ Vz $9^{*}$} & & $U$ & 2.64 & 0.74 & 167.2 & 7.8 & 2 & Vz 26 & & $U$ & 2.45 & 0.13 & 63.4 & 2.1 & 2 \\
\hline & & $B$ & 3.40 & 0.53 & 177.9 & 4.5 & & & & $B$ & 2.88 & 0.35 & 63.5 & 3.8 & \\
\hline & & $V$ & 5.36 & 1.24 & 31.8 & 6.5 & & & & V & 3.01 & 0.18 & 64.1 & 2.0 & \\
\hline & & $R$ & 4.35 & 0.58 & 42.8 & 3.8 & & & & $R$ & 2.73 & 0.22 & 61.2 & 2.2 & \\
\hline & & $I$ & 4.05 & 1.87 & 62.0 & 2.4 & & & & $I$ & 2.93 & 0.27 & 68.0 & 2.1 & \\
\hline \multirow[t]{5}{*}{ Vz $10^{*}$} & MV5 & $U$ & 3.92 & 3.75 & 6.4 & 7.5 & 2 & Vz 27 & & $U$ & 0.89 & 0.22 & 71.9 & 6.8 & 4 \\
\hline & & $B$ & 1.71 & 0.63 & 66.3 & 7.1 & & & & $B$ & 2.03 & 0.09 & 74.1 & 1.3 & \\
\hline & & $V$ & 2.18 & 0.11 & 53.0 & 1.4 & & & & V & 1.74 & 0.06 & 69.8 & 1.0 & \\
\hline & & $R$ & 2.37 & 0.07 & 56.7 & 0.9 & & & & $R$ & 1.75 & 0.07 & 69.9 & 1.2 & \\
\hline & & $I$ & 2.35 & 0.07 & 56.8 & 0.9 & & & & $I$ & 1.69 & 0.10 & 70.0 & 1.7 & \\
\hline \multirow{5}{*}{ Vz $11^{*}$} & & $U$ & 1.74 & 0.43 & 62.8 & 6.9 & 3 & Vz 28 & & $U$ & 1.38 & 0.10 & 73.8 & 2.1 & 2 \\
\hline & & $B$ & 2.78 & 0.13 & 62.8 & 1.3 & & & & $B$ & 1.18 & 0.05 & 76.8 & 1.3 & \\
\hline & & $V$ & 2.87 & 0.11 & 64.8 & 1.1 & & & & V & 1.26 & 0.05 & 70.1 & 1.0 & \\
\hline & & $R$ & 2.66 & 0.11 & 64.4 & 1.2 & & & & $R$ & 1.17 & 0.04 & 72.5 & 0.9 & \\
\hline & & $I$ & 2.43 & 0.13 & 64.3 & 1.5 & & & & $I$ & 1.21 & 0.04 & 71.0 & 0.9 & \\
\hline \multirow[t]{5}{*}{$\mathrm{Vz} 12^{*}$} & MV6 & $U$ & 2.78 & 0.23 & 65.9 & 2.7 & 4 & Vz $31^{*}$ & & $U$ & 2.38 & 0.98 & 65.6 & 11.2 & 4 \\
\hline & & $B$ & 2.95 & 0.21 & 66.2 & 1.9 & & & & $B$ & 2.94 & 0.36 & 59.6 & 3.5 & \\
\hline & & $V$ & 3.52 & 0.12 & 63.1 & 1.1 & & & & $V$ & 2.46 & 0.26 & 68.2 & 3.0 & \\
\hline & & $R$ & 3.67 & 0.12 & 60.4 & 1.1 & & & & $R$ & 2.69 & 0.20 & 73.3 & 2.1 & \\
\hline & & $I$ & 2.21 & 0.26 & 48.6 & 3.4 & & & & $I$ & 1.29 & 0.60 & 77.8 & 12.5 & \\
\hline Vz $13^{*}$ & MV8 & $U$ & 2.76 & 0.19 & 62.6 & 1.9 & 2 & Vz 33 & & $U$ & 1.83 & 0.53 & 66.4 & 8.1 & 4 \\
\hline & & $B$ & 3.69 & 0.18 & 64.8 & 1.4 & & & & $B$ & 1.95 & 0.09 & 70.7 & 1.3 & \\
\hline & & $V$ & 3.47 & 0.26 & 67.1 & 2.2 & & & & V & 2.38 & 0.18 & 70.5 & 2.2 & \\
\hline & & $R$ & 3.19 & 0.31 & 63.1 & 2.8 & & & & $R$ & 2.75 & 0.07 & 66.4 & 0.7 & \\
\hline & & $I$ & 3.39 & 0.20 & 68.1 & 5.1 & & & & $I$ & 3.27 & 0.13 & 64.9 & 1.2 & \\
\hline
\end{tabular}

Table 1. continued. 
A. M. Orsatti et al.: Revisiting the region of the open cluster NGC 5606, Online Material p 3

Table 1. continued.

\begin{tabular}{|c|c|c|c|c|c|c|c|c|c|c|c|c|c|c|c|}
\hline$\overline{\operatorname{Star}^{a}}$ & Other id. ${ }^{b}$ & Filter & $\begin{array}{l}P_{\lambda} \\
\%\end{array}$ & $\epsilon_{p}$ & $\begin{array}{c}\theta_{\lambda} \\
\circ\end{array}$ & $\epsilon_{\theta}$ & $\overline{n^{c}}$ & $\overline{\operatorname{Star}^{a}}$ & Other id. $^{b}$ & Filter & $\begin{array}{l}P_{\lambda} \\
\%\end{array}$ & $\epsilon_{p}$ & $\overline{\theta_{\lambda}}$ & $\epsilon_{\theta}$ & $\overline{n^{c}}$ \\
\hline \multirow[t]{5}{*}{ Vz 35} & & $\bar{U}$ & 1.47 & 0.46 & 64.4 & 8.7 & 3 & Vz 47 & & $\bar{U}$ & - & - & - & - & 4 \\
\hline & & $B$ & 2.03 & 0.09 & 70.7 & 1.3 & & & & $B$ & - & - & - & - & \\
\hline & & $V$ & 2.25 & 0.10 & 70.2 & 1.3 & & & & $V$ & 1.32 & 0.14 & 63.3 & 3.0 & \\
\hline & & $R$ & 1.97 & 0.10 & 69.6 & 1.5 & & & & $R$ & 1.38 & 0.07 & 65.3 & 1.5 & \\
\hline & & $I$ & 1.77 & 0.10 & 66.6 & 1.7 & & & & $I$ & 1.79 & 0.11 & 64.4 & 1.7 & \\
\hline \multirow[t]{5}{*}{ Vz $36^{*}$} & & $U$ & 2.55 & 0.18 & 64.4 & 2.0 & 2 & $\mathrm{Vz} 48$ & & $U$ & - & - & - & - & 4 \\
\hline & & $B$ & 3.58 & 0.17 & 64.4 & 1.4 & & & & $B$ & 2.03 & 0.14 & 66.6 & 1.9 & \\
\hline & & V & 3.05 & 0.10 & 65.0 & 0.9 & & & & V & 2.23 & 0.06 & 66.0 & 0.8 & \\
\hline & & $R$ & 3.13 & 0.10 & 63.1 & 0.9 & & & & $R$ & 1.90 & 0.04 & 67.1 & 0.7 & \\
\hline & & $I$ & 2.31 & 0.21 & 66.0 & 2.6 & & & & $I$ & 1.97 & 0.08 & 65.4 & 1.1 & \\
\hline \multirow[t]{5}{*}{ Vz 37} & & $U$ & 2.47 & 0.27 & 65.7 & 3.1 & 3 & Vz 49 & & $U$ & 0.55 & 0.35 & 33.9 & 16.1 & 4 \\
\hline & & $B$ & 1.72 & 0.09 & 74.3 & 1.5 & & & & $B$ & 1.74 & 0.27 & 74.3 & 4.5 & \\
\hline & & $V$ & 1.60 & 0.08 & 71.4 & 1.5 & & & & $V$ & 2.04 & 0.27 & 62.7 & 3.8 & \\
\hline & & $R$ & 1.63 & 0.03 & 67.7 & 0.6 & & & & $R$ & 2.17 & 0.23 & 64.5 & 3.0 & \\
\hline & & $I$ & 1.25 & 0.05 & 68.6 & 1.2 & & & & $I$ & 1.90 & 0.26 & 57.4 & 4.0 & \\
\hline \multirow[t]{5}{*}{ Vz $38^{*}$} & & $U$ & 1.05 & 0.22 & 78.6 & 5.9 & 4 & Vz 50 & & $U$ & 1.54 & 0.45 & 73.6 & 8.2 & 4 \\
\hline & & $B$ & 1.79 & 0.08 & 69.4 & 1.4 & & & & $B$ & 2.15 & 0.29 & 73.6 & 3.8 & \\
\hline & & $V$ & 2.47 & 0.18 & 61.5 & 2.1 & & & & V & 2.33 & 0.15 & 73.4 & 1.9 & \\
\hline & & $R$ & 2.59 & 0.40 & 66.7 & 4.4 & & & & $R$ & 2.32 & 0.09 & 70.8 & 1.1 & \\
\hline & & $I$ & 3.37 & 0.43 & 59.6 & 3.6 & & & & $I$ & 1.94 & 0.44 & 71.6 & 6.4 & \\
\hline \multirow[t]{5}{*}{ Vz 39} & & $U$ & 2.37 & 0.28 & 59.3 & 3.4 & 2 & Vz 52 & & $U$ & 3.85 & 0.32 & 114.6 & 2.3 & 3 \\
\hline & & $B$ & 1.93 & 0.19 & 71.0 & 2.8 & & & & $B$ & 2.65 & 0.21 & 72.2 & 2.3 & \\
\hline & & $V$ & 2.12 & 0.13 & 70.0 & 1.9 & & & & $V$ & 2.20 & 0.14 & 67.9 & 1.9 & \\
\hline & & $R$ & 1.99 & 0.10 & 71.5 & 1.4 & & & & $R$ & 2.11 & 0.23 & 70.8 & 3.0 & \\
\hline & & $I$ & 2.04 & 0.21 & 75.4 & 2.9 & & & & $I$ & 2.02 & 0.23 & 70.4 & 3.9 & \\
\hline \multirow[t]{5}{*}{$\mathrm{Vz} 40^{*}$} & & $U$ & 3.03 & 0.16 & 70.9 & 1.4 & 2 & Vz 53 & & $U$ & 3.76 & 0.32 & 67.0 & 3.5 & 4 \\
\hline & & $B$ & 3.08 & 0.04 & 70.2 & 1.2 & & & & $B$ & 2.04 & 0.14 & 69.8 & 1.9 & \\
\hline & & $V$ & 3.39 & 0.04 & 69.2 & 0.4 & & & & $V$ & 2.11 & 0.08 & 69.1 & 1.1 & \\
\hline & & $R$ & 3.27 & 0.08 & 69.9 & 0.7 & & & & $R$ & 2.09 & 0.13 & 71.0 & 1.8 & \\
\hline & & $I$ & 2.99 & 0.15 & 71.3 & 1.5 & & & & $I$ & 1.83 & 0.15 & 69.9 & 2.4 & \\
\hline \multirow[t]{5}{*}{ Vz 41} & & $U$ & - & - & - & - & 2 & Vz 54 & & $U$ & 2.88 & 0.78 & 83.2 & 7.5 & 3 \\
\hline & & $B$ & - & - & - & - & & & & $B$ & 2.52 & 0.13 & 75.3 & 1.5 & \\
\hline & & $V$ & 2.57 & 0.09 & 69.4 & 1.0 & & & & $V$ & 2.36 & 0.07 & 69.9 & 0.8 & \\
\hline & & $R$ & 3.15 & 0.11 & 71.5 & 1.0 & & & & $R$ & 2.07 & 0.07 & 67.1 & 1.0 & \\
\hline & & $I$ & 2.66 & 0.10 & 71.5 & 1.1 & & & & $I$ & 2.37 & 0.15 & 68.5 & 1.9 & \\
\hline \multirow[t]{5}{*}{ Vz 42} & & $U$ & 2.18 & 0.23 & 75.9 & 3.0 & 4 & Vz 55 & & $U$ & 5.51 & 1.75 & 66.2 & 8.8 & 6 \\
\hline & & $B$ & 1.60 & 0.17 & 7305 & 1.3 & & & & $B$ & 1.87 & 0.19 & 70.7 & 2.9 & \\
\hline & & $V$ & 1.53 & 0.09 & 76.4 & 1.9 & & & & $V$ & 1.67 & 0.19 & 71.6 & 3.2 & \\
\hline & & $R$ & 1.82 & 0.09 & 74.8 & 1.2 & & & & $R$ & 2.20 & 0.12 & 71.2 & 1.6 & \\
\hline & & $I$ & 1.20 & 0.15 & 72.2 & 1.3 & & & & $I$ & 1.88 & 0.17 & 72.3 & 2.6 & \\
\hline \multirow[t]{5}{*}{ Vz 43} & & $U$ & 1.46 & 0.17 & 76.1 & 3.3 & 4 & Vz 57 & MV12 & $U$ & 6.80 & 3.81 & 35.7 & 14.6 & 4 \\
\hline & & $B$ & 2.03 & 0.07 & 62.9 & 1.0 & & & & $B$ & 4.86 & 1.12 & 64.4 & 6.5 & \\
\hline & & $V$ & 2.24 & 0.05 & 71.7 & 0.6 & & & & V & 4.77 & 0.58 & 57.1 & 3.4 & \\
\hline & & $R$ & 1.96 & 0.08 & 70.5 & 1.2 & & & & $R$ & 4.59 & 0.62 & 58.9 & 3.8 & \\
\hline & & $I$ & 1.89 & 0.08 & 69.2 & 1.2 & & & & $I$ & 6.41 & 1.09 & 73.9 & 4.8 & \\
\hline \multirow[t]{5}{*}{ Vz 44} & & $U$ & 5.74 & 1.26 & 79.1 & 6.2 & 4 & Vz 58* & MV13 & $U$ & 2.49 & 0.25 & 68.4 & 2.9 & 4 \\
\hline & & $B$ & 2.40 & 0.23 & 73.1 & 2.8 & & & & $B$ & 2.67 & 0.25 & 63.9 & 2.6 & \\
\hline & & V & 1.90 & 0.08 & 69.0 & 1.2 & & & & V & 2.52 & 0.18 & 64.3 & 2.1 & \\
\hline & & $R$ & 2.19 & 0.08 & 73.4 & 1.0 & & & & $R$ & 2.97 & 0.14 & 61.2 & 1.5 & \\
\hline & & $I$ & 1.88 & 0.13 & 69.1 & 2.0 & & & & $I$ & 2.89 & 0.57 & 65.1 & 5.6 & \\
\hline \multirow[t]{5}{*}{ Vz 45} & & $U$ & - & - & - & - & 4 & Vz $59^{*}$ & MV11 & $U$ & 2.76 & 1.74 & 62.8 & 6.9 & 4 \\
\hline & & $B$ & - & - & - & - & & & & $B$ & 2.69 & 0.13 & 64.8 & 1.3 & \\
\hline & & $V$ & 1.02 & 0.09 & 66.1 & 2.4 & & & & $V$ & 2.79 & 0.11 & 64.8 & 1.1 & \\
\hline & & $R$ & 1.04 & 0.12 & 69.2 & 3.3 & & & & $R$ & 2.73 & 0.11 & 64.4 & 1.2 & \\
\hline & & $I$ & 1.09 & 0.13 & 59.0 & 3.3 & & & & $I$ & 2.75 & 0.13 & 64.3 & 1.5 & \\
\hline Vz 46 & & $U$ & 2.26 & 0.57 & 41.0 & 7.1 & 4 & Vz $60^{*}$ & & $U$ & 2.05 & 0.32 & 63.8 & 4.4 & 6 \\
\hline & & $B$ & 2.21 & 0.34 & 55.1 & 4.4 & & & & $B$ & 3.07 & 0.25 & 65.2 & 2.4 & \\
\hline & & $V$ & 2.14 & 0.25 & 72.0 & 3.3 & & & & $V$ & 3.01 & 0.15 & 66.8 & 1.4 & \\
\hline & & $R$ & 2.19 & 0.19 & 61.0 & 2.6 & & & & $R$ & 3.03 & 0.17 & 67.7 & 1.6 & \\
\hline & & $I$ & 2.67 & 0.80 & 48.5 & 8.3 & & & & $I$ & 2.72 & 0.40 & 54.3 & 4.2 & \\
\hline
\end{tabular}

Table 1. continued. 
A. M. Orsatti et al.: Revisiting the region of the open cluster NGC 5606, Online Material p 4

Table 1. continued.

\begin{tabular}{|c|c|c|c|c|c|c|c|}
\hline $\operatorname{Star}^{a}$ & Other id. ${ }^{b}$ & Filter & $\begin{array}{l}P_{\lambda} \\
\%\end{array}$ & $\epsilon_{p}$ & $\begin{array}{c}\theta_{\lambda} \\
\circ\end{array}$ & $\epsilon_{\theta}$ & $\overline{n^{c}}$ \\
\hline \multirow[t]{5}{*}{$\overline{\text { Vz } 61}$} & & $\bar{U}$ & 2.00 & 0.41 & 74.9 & 5.7 & 2 \\
\hline & & $B$ & 2.30 & 0.34 & 58.1 & 4.2 & \\
\hline & & $V$ & 2.99 & 0.13 & 61.2 & 1.3 & \\
\hline & & $R$ & 3.17 & 0.13 & 63.2 & 1.2 & \\
\hline & & $I$ & 4.40 & 0.29 & 59.7 & 1.9 & \\
\hline \multirow[t]{5}{*}{ Vz 63} & & $U$ & - & - & 117.2 & 29.9 & 4 \\
\hline & & $B$ & 2.04 & 1.35 & 58.2 & 16.7 & \\
\hline & & $V$ & 3.38 & 0.29 & 57.0 & 2.4 & \\
\hline & & $R$ & 3.68 & 0.70 & 68.6 & 5.4 & \\
\hline & & $I$ & 1.79 & 1.71 & 110.8 & 21.8 & \\
\hline \multirow[t]{5}{*}{ Vz 64} & & $U$ & 1.46 & 0.15 & 69.1 & 2.9 & 4 \\
\hline & & $B$ & 1.94 & 0.03 & 70.4 & 0.4 & \\
\hline & & $V$ & 2.11 & 0.03 & 70.7 & 0.3 & \\
\hline & & $R$ & 1.85 & 0.04 & 67.3 & 0.6 & \\
\hline & & $I$ & 1.66 & 0.05 & 67.4 & 0.9 & \\
\hline \multirow[t]{5}{*}{ Vz 65} & & $U$ & 1.28 & 0.16 & 64.9 & 3.6 & 4 \\
\hline & & $B$ & 1.39 & 0.04 & 69.1 & 0.7 & \\
\hline & & $V$ & 1.30 & 0.04 & 64.6 & 0.8 & \\
\hline & & $R$ & 1.39 & 0.04 & 68.9 & 0.9 & \\
\hline & & $I$ & 1.23 & 0.05 & 70.8 & 1.1 & \\
\hline \multirow[t]{5}{*}{ Vz 66} & & $U$ & 1.14 & 0.06 & 77.6 & 1.6 & 4 \\
\hline & & $B$ & 1.05 & 0.03 & 74.6 & 1.0 & \\
\hline & & $V$ & 1.29 & 0.03 & 76.0 & 0.7 & \\
\hline & & $R$ & 1.11 & 0.05 & 73.2 & 1.4 & \\
\hline & & $I$ & 1.01 & 0.06 & 73.7 & 1.7 & \\
\hline \multirow[t]{5}{*}{ Vz 68} & & $U$ & 2.65 & 0.51 & 71.4 & 5.5 & 4 \\
\hline & & $B$ & 1.64 & 0.12 & 61.2 & 2.0 & \\
\hline & & $V$ & 2.17 & 0.20 & 76.7 & 2.6 & \\
\hline & & $R$ & 1.67 & 0.27 & 76.9 & 4.6 & \\
\hline & & I & 2.11 & 0.33 & 60.0 & 4.5 & \\
\hline
\end{tabular}

${ }^{a}$ Identification from Vázquez \& Feinstein (1991); * stands for members in NGC 5606, according to them. ${ }^{b}$ Identifications from Moffat \& Vogt (1973). ${ }^{c}$ Number of integrations. 